\title{
Comparison among different diagnostic criteria for chronic ocular graft-versus-host disease applied with and without pre-transplant ophthalmological examination
}

\author{
Giuseppe Giannaccare $^{1} \cdot$ Piera Versura ${ }^{1} \cdot$ Francesca Bonifazi $\mathbb{D}^{2} \cdot$ Mariarosaria Sessa $^{2} \cdot$ Emilio C Campos ${ }^{1}$
}

Received: 31 July 2017 / Revised: 7 July 2018 / Accepted: 23 August 2018 / Published online: 7 September 2018

(c) The Royal College of Ophthalmologists 2018

\begin{abstract}
Purpose To compare the proportion and the agreement rate in the diagnosis of chronic ocular graft-versus-host disease (GVHD) among three criteria applied with and without ophthalmological examination before hematopoietic stem cell transplantation (HSCT).

Methods National Institutes of Health (NIH), International Consensus Criteria on ocular GVHD (ICCGVHD) and TFOS Dry Eye Workshop (DEWS) II criteria were used for the diagnosis of ocular GVHD according to two protocols: ophthalmological examination after HSCT (Protocol A), or before and after HSCT (Protocol B). Proportion of GVHD diagnosis and inter-rate agreement coefficient Kappa $(\mathrm{K})$ among the criteria were calculated.

Results One hundred nine patients undergone HSCT were included. NIH, ICCGVHD and DEWS II criteria diagnosed ocular GVHD in $14.7 \%, 17.4 \%$ and $59.6 \%$ of the patients (Protocol A), whereas in $11.9 \%, 15.6 \%$ and $33.0 \%$ of the HSCT patients (Protocol B). The coefficient K for the proportion of patients diagnosed with ocular GVHD by NIH and ICCGVHD criteria was $\mathrm{K}=0.626$ (Protocol A) and $\mathrm{K}=0.615$ (Protocol B). The $\mathrm{K}$ coefficient by NIH and DEWS II criteria was $\mathrm{K}=$ 0.144 (Protocol A), and $\mathrm{K}=0.233$ (Protocol B). The $\mathrm{K}$ coefficient by ICCGVHD and DEWS II criteria was $\mathrm{K}=0.250$ (Protocol A) and $\mathrm{K}=0.499$ (Protocol B). The $\mathrm{K}$ coefficient for ocular GVHD diagnosis applying Protocol A and B was $\mathrm{K}=$ 0.881 if NIH criteria were used, $\mathrm{K}=0.933$ if ICCGVHD criteria were used and $\mathrm{K}=0.501$ if DEWS II criteria were used. Conclusions The diagnosis of ocular GVHD varied significantly in our cohort of hematological patients according to both the diagnostic criteria used and the visit protocols applied.
\end{abstract}

\section{Introduction}

Allogeneic hematopoietic stem cell transplantation (HSCT) is currently the treatment of choice for a variety of malignant and non-malignant hematological disorders. Graftversus-host disease (GVHD) represents one of the major

These authors contributed equally: Giuseppe Giannaccare, Piera Versura.

Giuseppe Giannaccare

giuseppe.giannaccare@gmail.com

1 Ophthalmology Unit, DIMES, Alma Mater Studiorum, University of Bologna, S. Orsola- Malpighi University Hospital, Bologna, Italy

2 Haematology Institute 'L.A. Seragnoli', DIMES, Alma Mater Studiorum, University of Bologna, S. Orsola-Malpighi University Hospital, Bologna, Italy complications of HSCT, involving different tissues including the eye. Dry eye (DE) disease is the most frequent manifestation of ocular GVHD, and is reported to occur, to a various degree, in $40-76 \%$ of patients [1, 2]. Clinical features consist of conjunctival fibrosis, punctate and/or filamentous keratopathy, chronic blepharitis, atrophy and irregularity of the eyelid margin, which may eventually induce keratinization of the tarsal conjunctiva and symblepharon [3]. Symptoms include irritation, burning, foreign body sensation, pain, photophobia and blurred vision, with deeply impaired quality of life and daily activities [4].

Recently, the International TFOS Dry Eye Workshop (DEWS) II proposed a new comprehensive classification of overall DE disease, incorporating ocular discomfort symptoms, tear instability and osmolarity, and ocular surface staining [5].

Although there are no subjective symptoms or clinical signs pathognomonic of ocular GVHD, the National Institutes of Health $(\mathrm{NIH})$ introduced, and recently updated, 
specific criteria for its diagnosis, identifying the new onset of dry, gritty, or painful eyes, cicatricial conjunctivitis, keratoconjunctivitis sicca and confluent areas of punctate keratopathy as distinctive manifestations of chronic ocular GVHD [6, 7]. Later in 2013, the International Consensus Criteria on ocular GVHD (ICCGVHD) proposed a more detailed and specialized diagnosing and scoring schema, with the aim of replacing the NIH criteria [8].

However, the evidence of an ocular surface impairment in patients with hematological disorders already before HSCT is recently growing. In fact, the impairment of clinical tests along with morphological changes of meibomian glands with reduced gland areas detected by infrared meibography have been demonstrated in a large percentage of patients already prior to HSCT [9-12]. This evidence opens a new scenario, with the need for distinguishing between "conventional pre-existing dry eye" and "dry eye due to active ocular chronic GVHD." As a consequence, the ICCGVHD, the German-Austrian-Swiss Consensus Conference and the 2014 updated NIH Consensus Conference warranted a prospective study evaluating a comprehensive baseline pre-HSCT ophthalmological evaluation [7, 8, 13]. This new protocol could be relevant not only to reach early diagnosis and prompt treatment, but also to identify the real proportion of patients diagnosed as ocular GVHD after HSCT.

Therefore, the aim of the present study was to compare the proportion and the agreement rate in the diagnosis of chronic ocular GVHD among three different criteria, applied with and without pre-HSCT ophthalmological examination.

\section{Materials and methods}

\section{Study}

Patients who had undergone HSCT for various hematological disorders over the period March 2007-September 2015 at the Hematological Department of the S.OrsolaMalpighi University Hospital were screened for inclusion in this study. Ophthalmological and hematological data prospectively collected over the period March 2007 to March 2016 were reviewed. The study followed the tenets of the Declaration of Helsinki, and was approved by the local ethics committee of S.Orsola-Malpighi University Hospital. Each subject signed an informed consent before any study procedure. Inclusion criteria were: age above 18 years and the completeness of medical charts. Exclusion criteria were: history of previous ocular diseases, and the use of eye drops for the treatment of glaucoma. Conditioning regimen and HSCT procedure were performed as previously described [11]. Two scheduled ophthalmological visits had been performed, respectively, 7-9 days before HSCT and conditioning regimen (baseline, V0), and in a time window ranging from 3 to 6 months after HSCT (V1).

\section{Protocols}

Traditionally, the diagnosis of ocular GVHD has been conducted evaluating patients only after HSCT, thus reaching the diagnosis in the presence of post-HSCT ocular surface impairment, regardless the baseline pre-HSCT ocular conditions. For methodological purposes, in the present study, the protocol based only on post-HSCT ophthalmological examination was called "Protocol A". Recently, our and other groups proposed a new protocol including both pre- and post-HSCT ophthalmological assessment to proper diagnose and treat pre-existing $\mathrm{DE}$ before HSCT, as well as ocular GVHD after. In the present study, this protocol was called "Protocol B".

Furthermore, we compared the proportion of diagnosis of ocular GVHD and the agreement rate among three different classification criteria (NIH, ICCGVHD and TFOS DEWS II) by applying both Protocol A and Protocol B. This latter protocol, taking into account the baseline pre-HSCT ophthalmological evaluation, allows the identification of incident cases: those patients not suffering from DE before HSCT and developing it only after HSCT. The clarification of this issue, strongly suggested by NIH and ICCGVHD criteria, is crucial for a proper diagnosis of "new onset" of DE after HSCT, and thus for properly reaching the diagnosis of ocular GVHD. In this study, we identified as GVHD patients with new onset DE not only incident cases, but also those patients suffering from DE already before HSCT and worsening after (see below).

\section{GVHD diagnosis}

Chronic ocular GVHD diagnosis was reached according to three different classification criteria. Two of them, namely NIH [7] and ICCGVHD criteria [8], are already in use in this field. The third one is represented by our proposal to extend TFOS DEWS II criteria [5], currently used for conventional DE disease, to estimate also the diagnosis of ocular GVHD in hematological patients undergoing HSCT.

1. The NIH 2014 criteria identified as distinctive signs new ocular sicca with Schirmer's test values $\leq 5 \mathrm{~mm} / 5 \mathrm{~min}$ (preferably with confirmation of normal values at an established baseline), or a new onset of keratoconjunctivitis sicca by slit lamp examination with mean Schirmer's test values between 6 and $10 \mathrm{~mm} / 5 \mathrm{~min}$ (preferably with confirmation of normal values at an established baseline). These ocular signs were defined as distinctive, and an additional distinctive feature of at least another organ is 
Table 1 Grading scale according to National Institute of Health $(\mathrm{NIH})$ criteria [7]

\begin{tabular}{|c|c|c|c|c|}
\hline \\
\hline \multicolumn{5}{|l|}{ Diagnosis } \\
\hline \multicolumn{5}{|c|}{ - Schirmer test $\leq 5 \mathrm{~mm} / 5 \mathrm{~min}$} \\
\hline \multicolumn{5}{|l|}{ OR } \\
\hline \multicolumn{5}{|c|}{ - Schirmer test $6-10 \mathrm{~mm} / 5 \mathrm{~min}$ AND KCS by slit lamp examination } \\
\hline \multicolumn{5}{|l|}{ Severity grade } \\
\hline & Score 0 & Score 1 & Score 2 & Score 3 \\
\hline Symptoms & None & Mild & Moderate & Severe \\
\hline $\begin{array}{l}\text { Requirement of } \\
\text { lubricant eye drops }\end{array}$ & None & $\leq 3 \times$ day & $\begin{array}{l}>3 \times \text { day or } \\
\text { punctual plugs }\end{array}$ & Special eyewear to relieve pain \\
\hline ADL impairment & None & $\begin{array}{l}\text { Not } \\
\text { affected }\end{array}$ & Partially affected & $\begin{array}{l}\text { Significantly affected OR unable to } \\
\text { work OR loss of vision due to KCS }\end{array}$ \\
\hline
\end{tabular}

$K C S$ keratoconjunctivitis sicca, $A D L$ activities of daily living

\begin{tabular}{lllll}
\hline Severity scale & Schirmer test $(\mathrm{mm})$ & CFS (points) & OSDI (points) & Conjunctival injection \\
\hline 0 & $>15$ & 0 & $<13$ & None \\
1 & $11-15$ & $<2$ & $13-22$ & Mild/moderate \\
2 & $6-10$ & $2-3$ & $23-32$ & Severe \\
3 & $\leq 5$ & $\geq 4$ & $\geq 33$ & Severe \\
\hline Diagnosis & None (points) & Probable GVHD (points) & Definite GVHD (points) \\
\hline Systemic GVHD (-) & $0-5$ & $6-7$ & $\geq 8$ \\
Systemic GVHD (+) & $0-3$ & $4-5$ & $\geq 6$ \\
\hline
\end{tabular}

$C F S$ corneal fluorescein staining, $O S D I$ ocular surface disease index
Table 2 Grading scale according to the International Consensus Criteria on chronic ocular graft-versus-host disease (ICCGVHD) [8] considered necessary to reach the diagnosis for chronic ocular GVHD. The severity was assessed by a 4-point grade scale based on symptoms, requirement of lubricant eye drops and activities of daily living impairment (Table 1) [7]. The increase of at least 1 point of the severity scale after HSCT was considered as a worsening.

2. The ICCGVHD identified the following parameters for reaching the diagnosis of chronic ocular GVHD: ocular surface disease index (OSDI); Schirmer's test without anesthesia; corneal fluorescein staining; conjunctival injection. Severity scores from 0 to 3 were assigned to OSDI, corneal staining and Schirmer's values. Conjunctival injection was scored from 0 to 2 . Based on the presence or absence of systemic GVHD, definite ocular GVHD was reached, respectively, with at least 6 or 8 points (Table 2) [8]. The increase of at least 2 points of the score system after HSCT was considered as a worsening.

3. The TFOS DEWS II criteria required for the DE diagnosis, the presence of ocular discomfort symptoms, with an OSDI score $\geq 13$, and in addition, at least one eye had to exceed thresholds on one of the three subset signs, chosen among tear break-up time $($ TBUT $)<10$, tear osmolarity $\geq 308$, ocular surface staining $(>5$ corneal spots, $>9$ conjunctival spots, or lid wiper epitheliopathy of $\geq 2 \mathrm{~mm}$ in length and/or $\geq 25 \%$ sagittal width) (Table 3 ) [5]. The aggravation of at least one parameter among those
Table 3 Diagnostic criteria according to the TFOS DEWS II Diagnostic Methodology report [5]

Diagnosis

- Ocular Surface Discomfort Symptoms (OSDI $\geq 13)+1$ of

- TFBUT $<10 \mathrm{~s}$

- Tear osmolarity $\geq 308 \mathrm{mOsm} / \mathrm{L}$ in either eye or interocular difference $>8 \mathrm{mOsm} / \mathrm{L}$

- Ocular surface staining: $>5$ corneal spots, $>9$ conjunctival spots or lid margin ( $\geq 2 \mathrm{~mm}$ length and $\geq 25 \%$ width)

TFOS Tear Film and Ocular Surface Society, DEWS dry eye workshop, OSDI ocular surface disease index, TFBUT $(s)$ tear film break-up time (seconds)

studied after HSCT was considered as a worsening. According to our proposal, the diagnosis of ocular GVHD after HSCT can be reached in case of new positivity or worsening of TFOS DEWS II criteria, regardless of the involvement of other organs.

\section{Statistical analysis}

Descriptive statistics, including the median and range for continuous variables and the percentage and frequencies of categorical variables, were calculated for assessing the demographic and clinical characteristics of ocular GVHD 
Table 4 Demographic, clinical and HSCT-related characteristics of subjects included in the study

\begin{tabular}{|c|c|c|}
\hline & Patient number & $\%$ vs total \\
\hline \multicolumn{3}{|l|}{ Demographic data } \\
\hline Females & 54 & 49.5 \\
\hline Males & 55 & 50.5 \\
\hline Age (yrs) & $\begin{array}{l}46(18-70) \\
{[43-49]}\end{array}$ & \\
\hline \multicolumn{3}{|l|}{ Hematological history } \\
\hline \multicolumn{3}{|l|}{ Disorders } \\
\hline AML & 35 & 32.1 \\
\hline ALL & 22 & 20.1 \\
\hline HL & 10 & 9.1 \\
\hline CML & 10 & 9.1 \\
\hline NHL & 10 & 9.1 \\
\hline MM & 9 & 8.2 \\
\hline MDS & 7 & 6.2 \\
\hline CLL & 4 & 4.1 \\
\hline Other & 2 & 2.0 \\
\hline Time from diagnosis to HSCT (days) & $\begin{array}{l}290(110-2770) \\
{[261-494]}\end{array}$ & \\
\hline \multicolumn{3}{|l|}{ Disease stage } \\
\hline Early & 42 & 38.5 \\
\hline Advanced & 67 & 61.5 \\
\hline Previous autograft & 12 & 11.0 \\
\hline \multicolumn{3}{|l|}{$\begin{array}{l}\text { Previous chemotherapy medications } \\
\text { (no. cycles) }\end{array}$} \\
\hline$\leq 3$ cycles & 48 & 44.0 \\
\hline$>3$ cycles & 61 & 66.0 \\
\hline \multicolumn{3}{|l|}{ Ocular history } \\
\hline CL wearers & 19 & 17.4 \\
\hline VDT users & 15 & 13.7 \\
\hline Previous ocular surgery & 9 & 8.2 \\
\hline \multicolumn{3}{|l|}{ HSCT parameter } \\
\hline \multicolumn{3}{|l|}{ Donor characteristics } \\
\hline Age (yrs) & $\begin{array}{l}31.5(19-68) \\
{[27.9-33]}\end{array}$ & \\
\hline VUD & 86 & 78.8 \\
\hline HLA match & 26 & 28 \\
\hline HLA mismatch & 60 & 55.0 \\
\hline $\mathrm{RD}$ & 23 & 21.1 \\
\hline Sex mismatch & 52 & 47.7 \\
\hline \multicolumn{3}{|l|}{ Conditioning regimen } \\
\hline Reduced & 34 & 31.1 \\
\hline Myeloablative & 75 & 68.9 \\
\hline \multicolumn{3}{|l|}{ Stem cell source } \\
\hline Bone marrow & 45 & 41.2 \\
\hline Peripheral blood & 57 & 52.3 \\
\hline Cord blood & 7 & 6.5 \\
\hline GVHD history & & \\
\hline
\end{tabular}

Table 4 (continued)

\begin{tabular}{lll}
\hline & Patient number & $\%$ vs total \\
\hline Previous acute GVHD & & \\
aGVHD (+) & 49 & 45 \\
aGVHD (-) & 60 & 55 \\
Global assessment of aGVHD & & \\
(Glucksberg criteria) & 17 & 15.5 \\
Grade 1 & 23 & 21.2 \\
Grade 2 & 8 & 7.3 \\
Grade 3 & 1 & 0.9 \\
Grade 4 & & \\
Systemic chronic GVHD & 43 & 39.4 \\
cGVHD (+) & 66 & 60.6 \\
cGVHD (-) &
\end{tabular}

Data are expressed as median (min-max values) [95\% CI interval]

HSCT hematopoietic stem cell transplantation, ALL acute lymphoblastic leukemia, $A M L$ acute myeloid leukemia, $H L$ Hodgkin lymphoma, $C M L$ chronic myeloid leukemia, $N H L$ non-Hodgkin lymphoma, $M M$ multiple myeloma, $M D S$ myelodysplastic syndrome, $C L L$ chronic lymphocytic leukemia

patients. All data were analyzed with SPSS version 19.0 (SPSS Inc., Chicago, IL, USA).

The proportion of patients diagnosed with ocular GVHD and the relative $95 \%$ confidence interval $(95 \% \mathrm{CI})$ were calculated. Agreement of binary diagnoses between pairs of the three grading scales (NIH, ICCGVHD and TFOS DEWS II criteria) and of the two protocols (Protocols A and B) was evaluated with the Kappa coefficient, and its $95 \%$ CIs were estimated.

\section{Results}

One hundred nine Caucasian patients fulfilled the study criteria, and were included in the analysis. The characteristics of patients and HSCTs are summarized in Table 4. Sixty out of 109 patients (55\% of the total) were found to be affected by DE already before HSCT using TFOS DEWS II criteria.

The proportion of patients diagnosed with ocular GVHD according to the three criteria was analyzed as shown in Table 5. In particular, the NIH, the ICCGVHD and the DEWS II criteria diagnosed ocular GVHD in $14.7 \%, 17.4 \%$ and $59.6 \%$ of the HSCT patients, respectively, by applying Protocol A. Conversely, ocular GVHD was diagnosed in $11.9 \%, 15.6 \%$ and $33.0 \%$ of the HSCT patients, respectively, by applying Protocol B.

The inter-rate agreement coefficient Kappa $(\mathrm{K})$ for the proportion of patients diagnosed with ocular GVHD by NIH and ICCGVHD criteria was $\mathrm{K}=0.626 \quad(95 \% \quad \mathrm{CI}$ : $0.425-0.827$ ) when applying Protocol $\mathrm{A}$, and $\mathrm{K}=0.615$ 
Table 5 Proportion of patients diagnosed with ocular graft-versus-host disease (GVHD) according to different criteria and protocols used

\begin{tabular}{lllll}
\hline & Grading scale & Number & Proportion & $95 \%$ CI \\
\hline \multirow{2}{*}{ Protocol A } & TFOS DEWS II & & & \\
& Dry eye disease (+) & 65 & $59.6 \%$ & $0.502-0.689$ \\
& Dry eye disease (-) & 44 & $40.4 \%$ & $0.311-0.498$ \\
Protocol B & Dry eye disease (+) & 36 & $33.0 \%$ & $0.240-0.420$ \\
& Dry eye disease (-) & 73 & $67.0 \%$ & $0.580-0.760$ \\
& ICCGVHD & & & \\
Protocol A & Ocular GVHD (+) & 19 & $17.4 \%$ & $0.108-0.259$ \\
& Ocular GVHD (-) & 90 & $82.6 \%$ & $0.741-0.892$ \\
Protocol B & Ocular GVHD (+) & 17 & $15.6 \%$ & $0.094-0.238$ \\
& Ocular GVHD (-) & 92 & $84.4 \%$ & $0.762-0.906$ \\
& NIH & & & \\
Protocol A & Ocular GVHD (+) & 16 & $14.7 \%$ & $0.086-0.227$ \\
& Ocular GVHD (-) & 93 & $85.3 \%$ & $0.773-0.914$ \\
Protocol B & Ocular GVHD (+) & 13 & $11.9 \%$ & $0.065-0.195$ \\
& Ocular GVHD (-) & 96 & $88.1 \%$ & $0.805-0.935$ \\
\hline
\end{tabular}

TFOS Tear Film and Ocular Surface Society, DEWS dry eye workshop, ICCGVHD International Consensus Criteria on ocular GVHD, NIH National Institute of Health

(95\% CI: 0.398-0.831) when applying Protocol B. The $\mathrm{K}$ coefficient by $\mathrm{NIH}$ and TFOS DEWS II criteria was $\mathrm{K}=0.144$ (95\% CI: $0.040-0.248)$ when applying Protocol $\mathrm{A}$, and $\mathrm{K}=0.233$ (95\% CI: 0.059-0.407) when applying Protocol B. The K coefficient by ICCGVHD and TFOS DEWS II criteria was $\mathrm{K}=0.250$ (95\% CI: $0.140-0.360$ ) when applying Protocol $\mathrm{A}$, and $\mathrm{K}=0.499$ (95\% CI: $0.325-0.673$ ) when applying Protocol B.

The $\mathrm{K}$ coefficient for the proportion of patients diagnosed with ocular GVHD applying Protocol A and B was $\mathrm{K}=0.881$ (95\% CI: 0.750-1.012) if NIH criteria were used, $\mathrm{K}=0.933$ (95\% CI: 0.843-1.023) if ICCGVHD criteria were used, and $\mathrm{K}=0.501$ (95\% CI: 0.366-0.636) if DEWS II criteria were used.

\section{Discussion}

GVHD is a potentially severe complication that may develop after allogeneic HSCT, representing the most common cause of non-relapse mortality in hematological patients. The ocular surface is one of the target tissues of chronic GVHD, and about half of patients who undergo HSCT later develop DE, or experience its worsening. However, there are no specific symptoms, clinical signs or biomarkers available for a definite diagnosis of ocular GVHD. In fact, it mimics other diseases such as Sjögren or non-Sjögren-associated DE disease and blepharitis, making it difficult to definitively diagnose ocular GVHD, particularly in milder cases. Besides slit lamp examination and clinical tests, conjunctival biopsy is the only option for substantiating the diagnosis of ocular chronic GVHD [14].
However, biopsy is potentially harmful for these patients due to its invasiveness, and cannot be repeated indefinitely if disease monitoring is required.

Different diagnostic criteria for the assessment of the diagnosis and the severity of ocular GVHD have been introduced in the recent years [6-8, 13]. Among these, NIH and ICCGVHD criteria are the most commonly used in the field. In particular, the NIH eye score represents a part of a global assessment of chronic systemic GVHD for clinical trials, is easy to use and can be performed by the hematologists themselves. The severity grading has scientific limitations, being based on subjective and behavior-related criteria, and specific ophthalmologic examinations such as slit lamp examination and ocular tests other than Schirmer one, are not included in the recommendation. As a consequence, this classification appears far from satisfactory, and more recently the ICCGVHD proposed more detailed and specialized diagnostic criteria for chronic ocular GVHD, which need to be performed by an ophthalmologists. Both these two diagnostic criteria include Schirmer test, which represents a sole defining criterion in NIH criteria, or one of the four considered parameters in ICCGVHD criteria. However, Schirmer test is not specific, does not reflect the whole spectrum of the disease, and its reliability and sensitivity in diagnosing and monitoring $\mathrm{DE}$ have been shown to be very poor, particularly in milder cases $[15,16]$. Thus, it has been removed from the recently updated TFOS DEWS II criteria.

In addition, NIH and ICCGVHD criteria do not include TBUT, which is a diagnostic method for determining tear instability, or tear osmolarity, two well-recognized core mechanisms for DE, included in both definition and diagnostic criteria of TFOS DEWS II [17]. These criteria allow a more complete assessment of the tear film, which may provide deeper insight into the condition of the patient.

Recently, the evidence of ocular surface impairment in hematological patients already prior to HSCT is growing, with the need for a new protocol based on pre-HSCT ophthalmological examination, able to distinguish between "conventional pre-existing dry eye" and "dry eye due to active ocular chronic GVHD".

To overcome these inconsistencies, we applied TFOS DEWS II criteria, traditionally used in the overall DE disease and including also tear instability and osmolarity, to reach the diagnosis of ocular GVHD in our hematological population, and compared their diagnostic performance with NIH and ICCGVHD criteria, already in use in this field. As ocular GVHD seems to be an "organ-specific entity", we considered the positivity of TFOS DEWS II criteria sufficient to reach the diagnosis of ocular GVHD, irrespective of systemic GVHD status, as already proposed also by the current Chronic Ocular GVHD Workshop and the ICCGVHD criteria [8]. 
Two different protocols with (Protocol B) and without (Protocol A) the baseline pre-HSCT ophthalmological examination were applied to all the above mentioned criteria. The proportion of patients diagnosed as affected by ocular GVHD varied among the three criteria by applying both Protocols A and B. In particular, when Protocol A was used, over half of patients presented ocular GVHD according to TFOS DEWS II criteria, whereas the other two criteria reached the diagnosis in much less cases (about one-sixth). Instead, when Protocol B was applied, the proportion of ocular GVHD patients diagnosed by NIH and ICCGVHD criteria was similar (slightly lower), whereas that one obtained by TFOS DEWS II criteria was much lower (about half). The explanation for this difference is that TFOS DEWS II criteria diagnosed patients with hyperevaporative DE with low TBUT and pathological Meibomian gland dysfunction (MGD) scores but normal Schirmer test, whereas the other two criteria did not include this kind of patients because they did not investigate tear stability but only (NIH), or mainly (ICCGVHD), tear production. Therefore, the diagnostic performance of $\mathrm{NIH}$ and ICCGVHD criteria was not influenced by the type of protocol used, as they did not include among their parameters tear instability, which was demonstrated to be the main parameter impaired already before HSCT.

As a consequence, a high rate of disagreement among TFOS DEWS II and both ICCGVHD and NIH criteria was found by applying both protocols. However, this disagreement was higher between TFOS DEWS II and NIH, whereas was slightly lower between TFOS DEWS II and ICCGVHD because both these two criteria do not require the involvement of another organ to reach the diagnosis of ocular GVHD.

Our results appear consistent with a recent study, which reported substantial agreement between diagnosis reached by NIH and ICCGVHD criteria [18]. Another study compared the diagnostic accuracy of NIH eye score, the Japanese DE score and the DEWS 2007 score, and showed statistically good agreement among these criteria in the diagnostic rate and the severity of ocular GVHD [19]. However, it is difficult to compare our results with this work, because it was performed at a later stage of GVHD, and included a third scale (Japanese DE score), which was not analyzed in our study.

A recent study validated for the first time the ICCGVHD criteria, comparing them with best clinical practice (BCP), defined as ocular GVHD evaluation by highly trained single expert in ophthalmology on the basis of comprehensive clinical observation of the tear film and meibomian glands, among others [20]. There was slight overall agreement between BCP assessment and the ICCGVHD criteria for the diagnosis of chronic ocular GVHD. However, stratification by severity showed that
BCP was more sensitive in identifying patients with milder forms of ocular GVHD compared with the ICCGVHD criteria. The underestimation of the milder forms of GVHD by using ICCGVHD criteria is concordant with our results, and seems to be related to the exclusion of TBUT from the diagnostic criteria. On the contrary, the higher was the severity of ocular GVHD, the stronger was the association between BCP and ICCGVHD, reaching a full agreement among patients with severe ocular GVHD, in which also tear production is impaired and Schirmer test values are pathological.

Currently, tear instability is not included in the criteria in use in hematological patients, despite the increasing evidence on the impairment of the lids and meibomian glands even before and in the early stage following HSCT [12, 21-23]. In addition, the recent recommendations from the German-Austrian-Swiss Consensus Conference [13] and the GVHD Consortium for clinical trials [24] specifically targeting ocular GVHD agreed that other objective ophthalmological criteria with more detailed measures might be helpful to document ocular chronic GVHD activity. In fact almost the totality of the studies showed the poor performance of Schirmer test in the diagnosis of DE, and this test was recently removed in the NIH guidelines from the markers of severity and from the response criteria. In addition, as the Schirmer test score does not reflect changes in ocular GVHD activity, it was not recommended for the measurement of the changes in ocular GVHD studies by the Chronic GVHD Consortium.

In the present study, we did not measure tear osmolarity in all patients, and therefore we did not use this parameter to reach the diagnosis of DE disease according to TFOS DEWS II criteria. This issue represents the major weakness of the study.

In conclusion, the diagnosis of ocular GVHD varied significantly in our cohort of hematological patients not only according to the diagnostic criteria used, but also to the different visit protocols applied. In particular, the use of the Protocol B, including the baseline pretransplant ophthalmological examination, may allow to identify DE already prior to HSCT in the majority of patients, and thus to diagnose ocular GVHD after HSCT only in the presence of incident cases, i.e., patients not suffering from DE before HSCT and developing or worsening it after.

\section{Summary}

\section{What was known before}

- Ocular GVHD represents a major complication occurring after HSCT. Currently, different diagnostic criteria are in use in this setting for reaching ocular GVHD 
diagnosis. In addition, despite the recent evidence of ocular surface impairment already prior to HSCT, current criteria do not include baseline pre-HSCT ophthalmological examination.

\section{What this study adds}

- The diagnosis of ocular GVHD varied significantly in our cohort of hematological patients, not only according to the diagnostic criteria used, but also to the different visit protocols applied. Baseline ophthalmological examination may allow to identify DE already prior to HSCT, and thus to diagnose ocular GVHD after HSCT only in the presence of incident cases.

\section{Compliance with ethical standards}

Conflict of interest The authors declare that they have no conflict of interest.

\section{References}

1. Kim SK. Ocular graft vs. host disease. Ocul Surf. 2005;3:S177-9.

2. Hessen M, Akpek EK. Ocular graft-versus-host disease. Curr Opin Allergy Clin Immunol. 2012;12:540-7.

3. Busin M, Giannaccare G, Sapigni L, Testoni N, Leon P, Versura $\mathrm{P}$, et al. Conjunctival and limbal transplantation from the same living-related bone marrow donor to patients with severe ocular graft-vs-host disease. JAMA Ophthalmol. 2017;135:1123-5.

4. Westeneng AC, Hettinga Y, Lokhorst H, Verdonck L, van Dorp S, Rothova A. Ocular graft-versus-host disease after allogeneic stem cell transplantation. Cornea. 2010;29:758-63.

5. Wolffsohn JS, Arita R, Chalmers R, Djalilian A, Dogru M, Dumbleton K, et al. TFOS DEWS II diagnostic methodology report. Ocul Surf. 2017;15:539-74.

6. Filipovich AH, Weisdorf D, Pavletic S, Socie G, Wingard JR, Lee SJ, et al. National Institutes of Health consensus development project on criteria for clinical trials in chronic graft-versus-host disease: I. Diagnosis and staging working group report. Biol Blood Marrow Transplant. 2005;11:945-55.

7. Jagasia MH, Greinix HT, Arora M, Williams KM, Wolff D, Cowen EW, et al. National Institutes of Health consensus development project on criteria for clinical trials in chronic graft-versus-host disease: I. The diagnosis and staging working group report. Biol Blood Marrow Transplant. 2015;21:389-401.

8. Ogawa Y, Kim SK, Dana R, Clayton J, Jain S, Rosenblatt MI, et al. International chronic ocular graft-vs- host disease (GVHD) consensus group: proposed criteria for chronic GVHD (Part I). Sci Rep. 2013;3:3419. https://doi.org/10.1038/srep03419

9. Engel LA, Wittig S, Bock F, Sauerbier L, Scheid C, Holtick U, et al. Meibography and meibomian gland measurements in ocular graft-versus-host disease. Bone Marrow Transplant. 2015;50: 961-7.

10. Giannaccare G, Bonifazi F, Sessa M, Fresina M, Arpinati M, Bandini G, et al. Dry eye disease is already present in hematological patients before hematopoietic stem cell transplantation. Cornea. 2016;35:638-43.

11. Giannaccare G, Bonifazi F, Sessa M, Dan E, Arpinati M, Fresina $\mathrm{M}$, et al. Ocular surface analysis in hematological patients before and after allogeneic hematopoietic stem cell transplantation: implication for daily clinical practice. Eye (Lond). 2017;31: $1417-26$

12. Giannaccare G, Bonifazi F, Sebastiani S, Sessa M, Pellegrini M, Arpinati M, et al. Meibomian gland dropout in hematological patients prior to hematopoietic stem cell transplantation. Cornea. 2018. https://doi.org/10.1097/ICO.0000000000001585.

13. Dietrich-Ntoukas T, Cursiefen C, Westekemper H, Eberwein P, Reinhard T, Bertz $\mathrm{H}$, et al. Diagnosis and treatment of ocular chronic graft-versus-host disease: report from the GermanAustrian-Swiss Consensus Conference on Clinical Practice in Chronic GVHD. Cornea. 2012;31:299-310.

14. Eberwein P, Issleib S, Böhringer D, Mittelviefhaus H, Schwartzkopff J, Finke J, et al. Conjunctival HLA-DR and CD8 expression detected by impression cytology in ocular graft versus host disease. Mol Vis. 2013;19:1492-501.

15. Nichols KK, Mitchell GL, Zadnik K. The repeatability of clinical measurements of dry eye. Cornea. 2004;23:272-85.

16. Versura P, Frigato M, Cellini M, Mulè R, Malavolta N, Campos EC. Diagnostic performance of tear function tests in Sjogren's syndrome patients. Eye. 2007;21:229-37.

17. Craig JP, Nichols KK, Akpek EK, Caffery B, Dua HS, Joo CK, et al. TFOS DEWS II definition and classification report. Ocul Surf. 2017;5:276-83.

18. Pathak M, Diep PP, Lai X, Brinch L, Ruud E, Drolsum L. Ocular findings and ocular graft-versus-host disease after allogeneic stem cell transplantation without total body irradiation. Bone Marrow Transplant. 2018. https://doi.org/10.1038/s41409-018-0090-z.

19. Tatematsu Y, Ogawa Y, Abe T, Kamoi M, Uchino M, Saijo-Ban $\mathrm{Y}$, et al. Grading criteria for chronic ocular graft-versus-host disease: comparing the NIH eye score, Japanese dry eye score, and DEWS 2007 score. Sci Rep. 2014;4:6680. https://doi.org/10. 1038/srep06680.

20. Rapoport Y, Freeman T, Koyama T, Engelhardt BG, Jagasia M, Savani BN. Validation of International Chronic Ocular GraftVersus-Host Disease (GVHD) Group diagnostic criteria as a chronic ocular GVHD-specific metric. Cornea. 2017;36:258-63.

21. Wang Y, Ogawa Y, Dogru M, Tatematsu Y, Uchino M, Kamoi M. Baseline profiles of ocular surface and tear dynamics after allogeneic hematopoietic stem cell transplantation in patients with or without chronic GVHD-related dry eye. Bone Marrow Transplant. 2010;45:1077-83.

22. Kim S, Yoo YS, Kim HS, Joo CK, Na KS. Changes of meibomian glands in the early stage of post hematopoietic stem cell transplantation. Exp Eye Res. 2017;163:85-90.

23. Blecha C, Wolff D, Holler B, Holler E, Weber D, Vogt R. Verification of the new grading scale for ocular chronic graft-versushost disease developed by the German-Austrian-Swiss consensus conference on chronic GVHD. Ann Hematol. 2016;95:493-9.

24. Inamoto $\mathrm{Y}$, Chai $\mathrm{X}$, Kurland $\mathrm{BF}$, Cutler $\mathrm{C}$, Flowers ME, Palmer $\mathrm{JM}$, et al. Validation of measurement scales in ocular graft-versushost disease. Ophthalmology. 2012;119:487-93. 